\title{
SECOND ORDER CONNECTIONS
}

\author{
ROBERT H. BOWMAN
}

\section{Introduction}

The purpose of this paper is to investigate certain higher order structures on an $n$-dimensional $C^{\infty}$ manifold $M$, obtained by defining a connection on the bundle ${ }_{0}^{2} \Pi:{ }^{2} M \rightarrow M .{ }^{2} M$ has been called the second extension of $M$ by the present writer [1], and the second order tangent bundle by Yano and Ishihara [4].

We first define a second order connection on $M$ as a connection on ${ }_{0}^{2} \Pi:{ }^{2} M$ $\rightarrow M$ which induces a (first order) connection on $M$. It then follows that a second order connection of $M$ defines a unique vector bundle structure on ${ }_{0}^{2} \Pi:{ }^{2} M \rightarrow M$ and thus allows us to define the connection map [2], [3] of a second order connection. Covariant differentiation of a section of the vector bundle ${ }_{0}^{2} \Pi:{ }^{2} M \rightarrow M$ with respect to a vector field on $M$ is defined. This determines a concept of higher order parallelism and consequently two types of geodesics in $M$, which we call first and second order geodesics.

Given a (first order) connection on $M$ we show that it induces a second order connection on $M$, and that if the first order connection is linear, then so is the induced second order connection. The case of a second order connection induced from a linear (first order) connection is investigated in detail. We show that in this case the first order geodesics are the usual geodesics (of the first order connection) of $M$, and that there are second order geodesics of $M$ which are not first order geodesics. Using these second order geodesics we define a family of exponential maps and the related family of normal coordinates of $M$, which include the usual exponential map and normal coordinates as a special case.

We define the second order torsion tensor of a second order induced linear connection, and the second order Riemannian metric induced from a Riemannian metric on $M$. In the case that the first order connection is Riemannian we show that the induced second order connection is Riemannian with respect to the induced second order metric of the canonical first order metric. We define the second order curvature tensor of the induced linear second order connection, and the associated second order Riemann-Christoffel tensor, which may be used to define two natural invariants $K_{\mathrm{I}}$ and $K_{\mathrm{II}}$ which we call the Ist and IInd second order curvatures of $M$ respectively.

Communicated by K. Yano, May 10, 1971. 


\section{Notation and preliminary remarks}

Suppose that $M$ is an $n$-dimensional $C^{\infty}$ manifold, which we suppose is Hausdorff. Then we may form the tangent bundle ${ }_{0}^{1} \Pi: T M \rightarrow M$ of $M$ and the tangent bundle $T_{I I}: T T M \rightarrow T M$ of $T M$. From the commutative diagram

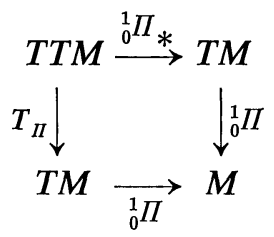

we see that $T T M$ is also a vector bundle under the tangent map ${ }_{0}^{1} \Pi_{*}$ of ${ }_{0}^{1} \Pi$. If we take

$$
{ }^{2} M=\left\{A \mid A \in T T M, T_{\Pi} A={ }_{0}^{1} \Pi_{*} A\right\},
$$

then ${ }^{2} M$ is an imbedded submanifold of $T T M$, and we have the sequence of $C^{\infty}$ manifolds and $C^{\infty}$ maps [1]

$$
{ }^{0} M \underset{{ }_{0}^{1} \Pi}{\longleftarrow}{ }^{1} M \underset{{ }_{1}^{2} \Pi}{\longleftarrow}{ }^{2} M
$$

where ${ }^{0} M=M,{ }^{1} M=T M$, and ${ }_{1}^{2} \Pi$ is the restriction of ${ }_{0}^{1} \Pi_{*}$ to ${ }^{2} M$, from which we see that both ${ }_{1}^{2} \Pi:{ }^{2} M \rightarrow T M$ and ${ }_{0}^{2} \Pi:{ }^{2} M \rightarrow M$, where ${ }_{0}^{2} \Pi={ }_{0}^{1} \Pi \circ{ }_{1}^{2} \Pi$, are fiber bundles.

If $(U, \phi)$ is a coordinate chart of $M$ with coordinate functions $x^{i}, i=1$, $\cdots, n$, then the partial derivatives $\partial / \partial x^{i}, i=1, \cdots, n$, form a basis of the tangent space $T M_{p}\left(\equiv M_{p}\right)$ at each point $p \in U$. Thus by employing the summation convention or lower case Latin indices, each $X_{p} \in{ }_{0}^{1} \Pi^{-1}(U)$ may be expressed in the form

$$
X_{p}=x^{1 i \partial} / \partial x^{i}(p)
$$

and hence ${ }_{0}^{1} \Pi^{-1}(U)$ becomes a chart of $T M$ with the $2 n$ coordinate functions $x^{0 i}=x^{i}, x^{1 i}=X\left(x^{i}\right)$. Repeating this process we obtain a set of $4 n$ coordinate functions on $T_{I I}^{-1} \circ{ }_{0}^{1} \Pi^{-1}(U) \subset T T M$; however the defining condition (2.2) of ${ }^{2} M$ implies that the second and third sets of $n$ coordinate functions are equal [1], and this determines on ${ }^{2} M \cap T_{\Pi}^{-1} \circ{ }_{0}^{1} \Pi^{-1}(U)$ a set of $3 n$ coordinate functions $x^{0 i}, x^{1 i}, x^{2 i}$. In each case these coordinates are called the coordinates induced by $(U, \phi)$, or simply the induced coordinates. We note that ${ }^{2} M$ is a fiber bundle but not a vector bundle relative to the induced coordinates.

\section{Second order connections}

Definition. A connection on the bundle ${ }_{0}^{2} \Pi:{ }^{2} M \rightarrow M$ is a $C^{\infty}$ left splitting $V$ of the exact sequence 


$$
0 \longrightarrow V^{2} M \stackrel{J}{\longrightarrow} T^{2} M \stackrel{\Pi^{\prime}}{\longrightarrow}{ }_{0}^{2} \Pi^{-1} T M \longrightarrow 0 .
$$

Here ${ }_{0}^{2} \Pi^{-1} T M$ denotes the pull-back of $T M, \Pi^{\prime}$ the natural map defined by the tangent map ${ }_{0}^{2} \Pi_{*}$, and $J$ the inclusion map. In terms of the induced coordinates on ${ }^{2} M$, (3.1) becomes locally

$$
\begin{aligned}
0 \longrightarrow\left(x^{0}, x^{1}, x^{2} ; 0, y^{1}, y^{2}\right) \stackrel{J}{\longrightarrow}\left(x^{0}, x^{1}, x^{2} ; y^{0}, y^{1}, y^{2}\right) \\
\stackrel{\Pi^{\prime}}{\longrightarrow}\left(x^{0}, x^{1}, x^{2} ; y^{0}\right) \longrightarrow 0 .
\end{aligned}
$$

Since $V J=I d$, the identity on $V^{2} M$, we see that $V\left(x^{0}, x^{1}, x^{2} ; 0, y^{1}, y^{2}\right)=$ $\left(x^{0}, x^{1}, x^{2} ; 0, y^{1}, y^{2}\right)$ and hence that

$$
\begin{aligned}
& V\left(x^{0}, x^{1}, x^{2} ; y^{0}, y^{1}, y^{2}\right) \\
& \quad=\left(x^{0}, x^{1}, x^{2} ; 0, y^{1}+\omega^{1}\left(x^{0}, x^{1}, x^{2}\right) y^{0}, y^{2}+\omega^{2}\left(x^{0}, x^{1}, x^{2}\right) y^{0}\right),
\end{aligned}
$$

where $\omega^{1}$ and $\omega^{2}$ are the local components of the connection.

Definition. A connection on ${ }_{0}^{2} \Pi:{ }^{2} M \rightarrow M$ is a second order connection on $M$ provided that there exists a $C^{\infty}$ left splitting $\tilde{V}$ of

$$
0 \rightarrow V T M \rightarrow T T M \rightarrow{ }_{0}^{1} \Pi^{-1} T M \rightarrow 0
$$

such that

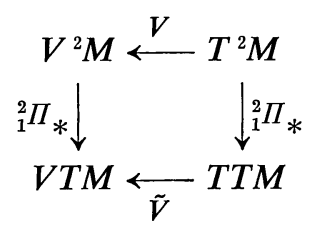

commutes. In terms of induced coordinates this means the local components of connection satisfy the condition

$$
\omega^{1}\left(x^{0}, x^{1}, x^{2}\right)=\tilde{\omega}\left(x^{0}, x^{1}\right),
$$

i.e., $\omega^{1}$ is independent of $x^{2}$, whence

$$
\begin{aligned}
& V\left(x^{0}, x^{1}, x^{2} ; y^{0}, y^{1}, y^{2}\right) \\
& \quad=\left(x^{0}, x^{1}, x^{2} ; 0, y^{1}+\omega^{1}\left(x^{0}, x^{1}\right) y^{0}, y^{2}+\omega^{2}\left(x^{0}, x^{1}, x^{2}\right) y^{0}\right) .
\end{aligned}
$$

We call $\tilde{V}$ the (first order) connection on $M$ induced by the second order connection $V$.

Theorem 3.1. Each second order connection on $M$ determines a unique vector bundle structure on ${ }_{0}^{2} \Pi:{ }^{2} M \rightarrow M$ such that with each chart of $M$ there is associated a vector bundle chart of ${ }^{2} M$. Moreover, this vector bundle 
structure allows us to define the connection map of the given second order connection.

Proof. Let $\tilde{D}: T T M \rightarrow T M$ be the connection map of the connection $\tilde{V}$, (this means that $\tilde{D}: V T M \rightarrow T M$ is an isomorphism determined by $\tilde{V}$ ) [2] [3]. Then $T T M$ has the unique vector bundle structure over $M$ determined by the diffeomorphism [6]

$$
{ }_{0}^{1} \Pi_{*} \oplus \tilde{D} \oplus T_{\Pi}: T T M \rightarrow T M \oplus T M \oplus T M \quad \text { (Whitney Sum) }
$$

and is denoted by $\sigma: T T M \rightarrow M$.

If $(U, \phi)$ is a chart of $M$, then the induced chart in $T M$ determines a vector bundle chart (or $V B$-chart) via (3.6). Since ${ }^{2} M$ is the imbedded submanifold of TTM consisting of points $A$ such that ${ }_{0}^{1} \Pi_{*} A=T_{\Pi} A$ we see that if

$$
\Delta: T M \oplus T M \rightarrow T M \oplus T M \oplus T M
$$

is the natural isomorphism of $T M \oplus T M$ onto ${ }_{0}^{1} \Pi_{*} \oplus \tilde{D} \oplus T_{I I}\left({ }^{2} M\right)$, then

$$
\Delta^{-1} \circ\left({ }_{0}^{1} \Pi_{*} \oplus \tilde{D} \oplus T_{I}\left({ }^{2} M\right)\right)={ }_{0}^{1} \Pi_{*} \oplus \tilde{D}\left({ }^{2} M\right),
$$

and so we may give ${ }^{2} M$ the structure of a vector bundle over $M$ via the diffeomorphism

$$
{ }_{0}^{1} \Pi_{*} \oplus \tilde{D}:{ }^{2} M \rightarrow T M \oplus T M
$$

which determines a $V B$-chart on ${ }^{2} M$ for each chart of $M$.

If $A \in T T M$, then in terms of the induced coordinates $A=A^{0 i} \partial_{0 i}+A^{1 i} \partial_{1 i}$ where $\partial_{0 i}=\partial / \partial x^{i}, \partial_{1 i}=\partial / \partial x^{1 i}$, and we have

$$
\tilde{D}(A)=\left(A^{1 i}+\omega_{j}^{1 i}\left(x^{0}, x^{1}\right) A^{0 j}\right) \partial_{0 i},
$$

so that if we take

$$
X_{i}^{h}=\partial_{0 i}-\omega_{i j}^{1 j} \partial_{1 j}, \quad X_{i}^{v}=\partial_{1 i},
$$

we see that

$$
\begin{array}{ll}
{ }_{0}^{1} \Pi_{*}\left(X_{i}^{h}\right)=\partial_{0 i}, & { }_{0}^{1} \Pi_{*}\left(X_{i}^{v}\right)=0, \\
\tilde{D}\left(X_{i}^{h}\right)=0, & \tilde{D}\left(X_{i}^{v}\right)=\partial_{0 i} .
\end{array}
$$

Now if $A \in{ }^{2} M$, we have ${ }_{0}^{1} \Pi_{*} A=T_{\Pi} A$, so that

$$
\begin{aligned}
A & =x^{1 i} \partial_{0 i}+A^{1 i} \partial_{1 i}=x^{1 i} \partial_{0 i}-x^{1 i} \omega_{i j}^{1 j} \partial_{1 j}+x^{1 i} \omega_{i}^{1 j} \partial_{1 j}+A^{1 i} \partial_{1 i} \\
& =x^{1 i} X_{i}^{h}+\left(A^{1 j}+x^{1 i} \omega_{i}^{1 j}\right) X_{j}^{v} .
\end{aligned}
$$

Thus we see that if $\left(x^{0}, x^{1}, x^{2}\right)$ are the coordinates of ${ }^{2} M$ induced from a given chart of $M$, then the associated $V B$-coordinates on ${ }^{2} M$ are 


$$
z^{0 i}=x^{0 i}, \quad z^{1 i}=x^{1 i}, \quad z^{2 i}=x^{2 i}+x^{1 j} \omega_{j}^{1 i}\left(x^{0}, x^{1}\right) .
$$

Since ${ }_{0}^{2} I\left(z^{0}, z^{1}, z^{2}\right)=z^{0}$, we see that the exact sequence (3.1) once again has the form (3.2) in terms of the $V B$-coordinates:

$$
\begin{aligned}
0 \rightarrow\left(z^{0}, z^{1}, z^{2} ; 0, v^{1}, v^{2}\right) \rightarrow\left(z^{0}, z^{1}, z^{2} ; v^{0}, v^{1}, v^{2}\right) & \\
& \rightarrow\left(z^{0}, z^{1}, z^{2} ; v^{0}\right) \rightarrow 0,
\end{aligned}
$$

and

$$
\begin{aligned}
& V\left(z^{0}, z^{1}, z^{2} ; v^{0}, v^{1}, v^{2}\right) \\
& \quad=\left(z^{0}, z^{1}, z^{2} ; 0, v^{1}+\omega^{1}\left(z^{0}, z^{1}\right) V^{0}, v^{2}+\omega^{2}\left(z^{0}, z^{1}, z^{2}\right) v^{0}\right)
\end{aligned}
$$

where $\omega^{1}$ and $\omega^{2}$ are the local components of the second order connection relative to the $V B$-coordinates, although we will use the same symbol as before.

Since ${ }^{2} M$ is a vector bundle relative to the $V B$-coordinates, there is connection map $D$ of the second order connection. This map is obtained in the usual manner by identifying $V^{2} M$ with ${ }_{0}^{2} \Pi^{-1}{ }^{2} M$ (the pull-back of ${ }^{2} M$ over itself) and applying the natural map of the pull-back which takes ${ }_{0}^{2} \Pi^{-12} M$ onto ${ }^{2} M$. It is necessary that ${ }^{2} M$ be a vector bundle in order that this be possible.

In terms of $V B$-coordinates the connection map $D$ becomes

$$
D\left(z^{0}, z^{1}, z^{2} ; y^{0}, y^{1}, y^{2}\right)=\left(z^{0}, y^{1}+\omega^{1}\left(z^{0}, z^{1}\right) y^{0}, y^{2}+\omega^{2}\left(z^{0}, z^{1}, z^{2}\right) y^{0}\right) .
$$

If $B \in T^{2} M$, then $B=B^{0 i \partial} / \partial z^{0 i}+B^{1 i} \partial / \partial z^{1 i}+B^{2 i} \partial / \partial z^{2 i}$, and thus for $D$ we have

(3.11) $\quad D(B)=\left(B^{1 i}+\omega_{j}^{1 i}\left(z^{0}, z^{1}\right) B^{0 j}\right) X_{i}^{h}+\left(B^{2 i}+\omega_{j}^{2 i}\left(z^{0}, z^{1}, z^{2}\right) B^{0 j}\right) X_{i}^{v}$.

If $A: M \rightarrow{ }^{2} M$ is a $C^{\infty}$ section of the vector bundle ${ }_{0}^{2} \Pi:{ }^{2} M \rightarrow M$, and $X$ a vector field on $M$, then the covariant derivative of $A$ with respect to $X$ is defined to be

$$
D_{X} A=D A_{*} X .
$$

If $X=\xi^{i} \partial_{i}$, and $A=A^{0 i} X_{i}^{h}+A^{1 i} X_{i}^{v}$ locally, then we have the local expression

$$
\begin{aligned}
D_{X} A= & \xi^{j}\left(\partial A^{0 i} / \partial z^{0 j}+\omega_{j}^{1 i}\left(z^{0}, A^{0}\right)\right) X_{i}^{h} \\
& +\xi^{j}\left(\partial A^{1 i} / \partial z^{0 j}+\omega_{j}^{2 i}\left(z^{0}, A^{0}, A^{1}\right)\right) X_{i}^{v} .
\end{aligned}
$$

If ${ }_{0}^{1} \Pi_{*} D_{X} A=0$, we will say that $A$ is first order parallel with respect to $X$; if $\tilde{D} D_{X} A=0$, we will say that $A$ is second order parallel with respect to $X$; and if both of these conditions are satisfied, we will say that $A$ is parallel with respect to $X$. 
If $\gamma: I \rightarrow M$ is a $C^{\infty}$ curve, then its canonical lift is the section $\gamma^{\prime}$ of $T M$ over $\gamma$ obtained by associating with each point $\gamma(t)$ the tangent vector to $\gamma$ at that point. Similarly we associate with each point $\gamma^{\prime}(t)$ its tangent vector in $T T M$ to obtain the second canonical lift $\gamma^{\prime \prime}$. In terms of local coordinates we see that if $x^{i}(t)=x^{i} \circ \gamma(t)$, then $\gamma^{\prime}(t)=\left(x(t), x^{\prime}(t)\right)$ and $\gamma^{\prime \prime}(t)=$ $\left(x(t), x^{\prime}(t), x^{\prime}(t), x^{\prime \prime}(t)\right)$. Thus we see that $\gamma^{\prime \prime}(t) \subset{ }^{2} M$, and hence it may be defined by the coordinates

$$
\begin{aligned}
& x^{0 i}=x^{i}(t), \quad x^{1 i}=x^{i^{\prime}}(t), \quad x^{2 i}=x^{i^{\prime \prime}}(t), \quad \text { or } \\
& z^{0 i}=x^{i}(t), \quad z^{1 i}=x^{i^{\prime}}(t), \quad z^{2 i}=x^{i^{\prime \prime}}(t)+\omega_{j}^{1 i}\left(x, x^{\prime}\right) x^{j^{\prime}}(t),
\end{aligned}
$$

where the $x$ 's are induced coordinates, and the $z$ 's are the associated $V B$ coordinates. From (3.12) we have

$$
D_{\gamma^{\prime}} \gamma^{\prime \prime}=\left(x^{i^{\prime \prime}}+\omega_{j}^{1 i}\left(x, x^{\prime}\right) x^{j^{\prime}}\right) X_{i}^{h}+\left(z^{2 i^{\prime}}+\omega_{j}^{2 i}\left(x, x^{\prime}, z^{2}\right) x^{j^{\prime}}\right) X_{i}^{v} .
$$

We will say that $\gamma$ is a first order geodesic provided that ${ }_{0}^{1} \Pi_{*} D_{\gamma^{\prime}} \gamma^{\prime \prime}=0$, and that $\gamma$ is a second order geodesic if $\tilde{D} D_{\gamma^{\prime}} \gamma^{\prime \prime}=0$.

\section{Higher order geodesics}

If we choose a coordinate chart $(U, \phi)$ of $M$ containing a point $p$, then we may define a local isomorphism of the vector bundle ${ }_{0}^{2} \Pi \mid{ }_{0}^{2} \Pi^{-1}(U):{ }_{0}^{2} \Pi^{-1}(U) \rightarrow$ $M$ into the vector bundle $\sigma: T T M \rightarrow M$ by

$$
I\left(z^{0} ; z^{1}, z^{2}\right)=\left(z^{0} ; 0, z^{1}, z^{2}\right) .
$$

Since this is independent of the chart chosen, there is then defined a global map

$$
I:{ }^{2} M \rightarrow T T M
$$

which locally has the form (4.1). Then we obtain the tangent map

$$
I_{*}: T^{2} M \rightarrow T T M
$$

which has the local form

$$
I_{*}\left(z^{0}, z^{1}, z^{2} ; y^{0}, y^{1}, y^{2}\right)=\left(z^{0}, 0, z^{1}, z^{2} ; y^{0}, 0, y^{1}, y^{2}\right) .
$$

Let $S$ be the symmetry map $S: T T M \rightarrow T T M$, [3].

Theorem 4.1. Each (first order) connection on $M$ induces a unique second order connection on $M$ whose connection map is given by $I^{-1} S \tilde{D}_{*} S S_{*} I_{*}$ and whose left splitting map is $\left(S S_{*} I\right)^{-1} \tilde{V}_{*}\left(S S_{*} I_{*}\right)$.

Proof. We first note that a (first order) connection on $M$ may be lifted to a connection (called the complete lift in [5]) on $T_{\Pi}: T T M \rightarrow T M$ with left 
splitting map $\hat{V}=\left(S S_{*}\right)^{-1} \tilde{V}_{*}\left(S S_{*}\right)$ and connection map $\hat{D}=S \tilde{D}_{*} S S_{*}$, [3]. Locally $D$ has the form

$$
D(a, b, c, d ; \alpha, \beta, \gamma, \delta)=\left(a, b, \gamma+\omega(a, c) \alpha, \delta+\omega(a, c) \beta+\omega^{\prime}(a, c)(b, d) \alpha\right),
$$

where $\omega(a, c)$ is the local component of the first order connection, and $\omega^{\prime}(a, c)$ its derivative. Thus, if we define

$$
D=I^{-1} \hat{D} I_{*},
$$

we have

$$
\begin{aligned}
D\left(z^{0}, z^{1}, z^{2} ; y^{0}, y^{1}, y^{2}\right) & =I^{-1} \hat{D}\left(z^{0}, 0, z^{1}, z^{2} ; y^{0}, 0, y^{1}, y^{2}\right) \\
& =I^{-1}\left(z^{0}, 0, y^{1}+\omega\left(z^{0}, z^{1}\right) y^{0}, y^{2}+\omega^{\prime}\left(z^{0}, z^{1}\right)\left(0, z^{2}\right) y^{0}\right) \\
& =\left(z^{0}, y^{1}+\omega\left(z^{0}, z^{1}\right) y^{0}, y^{2}+\omega^{\prime}\left(z^{0}, z^{1}\right)\left(0, z^{2}\right) y^{0}\right)
\end{aligned}
$$

which we see is a second order connection on $M$. That the left splitting map has the required form may be seen by a similar calculation using

$$
\begin{aligned}
& \left(S S_{*}\right)^{-1} \tilde{V}^{*}\left(S S_{*}\right)(a, b, c, d ; \alpha, \beta, \gamma, \delta) \\
& \quad=\left(a, b, c, d ; 0,0, \gamma+\omega(a, c) \alpha, \delta+\omega(a, c) \beta+\omega^{\prime}(a, c)(b, d) \alpha\right) .
\end{aligned}
$$

If $D$ is linear on the ${ }_{0}^{2} \Pi_{*}$ fibers of ${ }_{0}^{2} \Pi_{*}: T^{2} M \rightarrow T M$, then we will say that the associated second order connection, which we will henceforth also denote by $D$, is linear. In terms of $V B$-coordinates this means that

$$
\omega^{1}\left(z^{0}, z^{1}\right) y^{0}=\Gamma^{1}\left(z^{0}\right)\left(z^{1}, y^{0}\right), \quad \omega^{2}\left(z^{0}, z^{1}, z^{2}\right) y^{0}=\Gamma^{2}\left(z^{0}\right)\left(\left(z^{1}, z^{2}\right), y^{0}\right) .
$$

This may be seen by noting that $\omega^{1}\left(z^{0}, \ldots\right)$ is a linear map $R^{n} \rightarrow L\left(R^{n}, R^{n}\right)$ for each $z^{0} \in U$, and is thus an element of $L\left(R^{n}, L\left(R^{n}, R^{n}\right)\right) \approx L^{2}\left(R^{n}, R^{n}\right)$. The corresponding $\Gamma: U \rightarrow L^{2}\left(R^{n}, R^{n}\right)$ (under this topological isomorphism) is the local component of the linear connection. If $\omega$ is the local component of a linear (first order) connection on $M$, then

$$
\omega(a, b) c=\Gamma(a)(b, c)
$$

as above. Since

$$
\omega^{\prime}(a, b)(c, d) e=D_{1} \omega(a, b)(c, e)+D_{2} \omega(a, b)(d, e),
$$

where $D_{1}$ and $D_{2}$ are the partial derivatives with respect to the first and second variables respectively, and

$$
D_{1} \omega(a, b)(c, e)=\Gamma^{\prime}(a)(b, c, e), \quad D_{2} \omega(a, b)(d, e)=\Gamma(a)(d, e),
$$

we have 


$$
\omega^{\prime}(a, b)(c, d) e=\Gamma^{\prime}(a)(b, c, e)+\Gamma(a)(d, e) .
$$

Upon using (4.6) in (4.4) we see that

$$
D\left(z^{0}, z^{1}, z^{2} ; y^{0}, y^{1}, y^{2}\right)=\left(z^{0}, y^{1}+\Gamma\left(z^{0}\right)\left(z^{1}, y^{0}\right), y^{2}+\Gamma\left(z^{0}\right)\left(z^{2}, y^{0}\right)\right)
$$

and we have the following corollary to Theorem 4.1.

Corollary 4.1. Each (first order) linear connection on $M$ induces a second order connection on $M$, which is also linear and has the form (4.7).

Theorem 4.2. If $D$ is a second order connection on $M$ induced from a linear (first order) connection $\tilde{D}$ on $M$, then the geodesics of $\tilde{D}$ are geodesics of $D$. However, there are geodesics of $D$ which are not geodesic of $\tilde{D}$.

Proof. In the case of a second order connection induced from a linear (first order) connection we see from (3.14) that the equations of a first order geodesic in $M$ become

$$
x^{i^{\prime \prime}}+\Gamma_{j k}^{i} x^{j^{\prime}} x^{k^{\prime}}=0,
$$

which are the classical equations for a geodesic in $M$ relative to the first order connection. Also from (3.14) we see that the equations of a second order geódesic are

$$
z^{2 i^{\prime}}+\Gamma_{j k}^{i} x^{j^{\prime}} z^{2 k}=0, \quad z^{2 i}=x^{i^{\prime \prime}}+\Gamma_{j k}^{i} x^{j^{\prime}} x^{k^{\prime}},
$$

which are equivalent to

$$
\begin{aligned}
x^{i^{\prime \prime \prime}} & +\Gamma_{j k}^{i} x^{j^{\prime \prime}} x^{k^{\prime}}+2 \Gamma_{j k}^{i} x^{j^{\prime}} x^{k^{\prime \prime}}+\Gamma_{j k}^{i} \Gamma_{l m}^{k} x^{j^{\prime}} x^{l^{\prime}} x^{m^{\prime}} \\
& +\left(\partial \Gamma_{j k}^{i} / \partial x^{l}\right) x^{j^{\prime}} x^{k^{\prime}} x^{l^{\prime}}=0 .
\end{aligned}
$$

From (4.10) we see that for any set of initial conditions $\left(x^{i}\left(t_{0}\right), x^{i^{\prime}}\left(t_{0}\right), x^{i^{\prime \prime}}\left(t_{0}\right)\right)$ (alternately $\left(x^{i}\left(t_{0}\right), x^{i^{\prime}}\left(t_{0}\right), z^{2 i}\left(t_{0}\right)\right)$ there exists a unique solution satisfying these initial conditions. From (4.9) we see that if $z^{2 i}\left(t_{0}\right)=0$, then the corresponding solution to these equations is $z^{2 i}(t)=0$, and this condition is satisfied if and only if $x^{i}(t)$ is a first order geodesic. Hence each first order geodesic of $M$ is also a second order geodesic of $M$. On the other hand, if $z^{2 i}\left(t_{0}\right) \neq 0$, then $z^{2 i}(t) \neq 0$, and there are second order geodesics of $M$, which are not first order geodesics.

Theorem 4.3. Suppose that $D$ is a second order connection on $M$ induced from a linear (first order) connection on $M$. Then for each pair consisting of a vector $A \in T M_{p}$ and a 3-covariant $T M_{p}$-valued tensor $T$ at $p$, there corresponds an exponential map (depending on D) which maps a neighborhood of 0 in $\mathrm{TM}_{p}$ diffeomorphically onto a neighborhood of $p$ in $M$. If $A=0$, this is the usual exponential map (of the first order connection).

Proof. From the form of the differential equations (4.10) we see that if $\gamma_{(X, A)}$ is the second order geodesic through a point $p$ with initial conditions $x(0)=p, x^{\prime}(0)=X, z^{2}(0)=A$, then 


$$
\gamma_{(X, A)}(t)=\gamma_{(t X, t 2 A)}(1)
$$

when $\gamma_{(X, A)}(t)$ is defined. Take

$$
\operatorname{Exp}_{(p, T)}(X, A)=\gamma_{(X, T(A, X, X))}(1),
$$

when the right hand side is defined. From (4.11) we see that if $t \neq 0$, then

$$
\gamma_{(X, T(A, X, X))}(1)=\gamma_{(X / t, T(A, X / t, X / t))}(t),
$$

and thus for any $A$ we see that if $X$ is sufficiently small, then $\operatorname{Exp}_{(p, T)}(X, A)$ is defined. Suppose that $I_{A}: X \rightarrow(X, A)$, and define $\exp _{(p, T, A)}: T M_{p} \rightarrow M$ by

$$
\exp _{(p, T, A)}(X)=\operatorname{Exp}_{(p, T)} \circ I_{A}(X)
$$

If $\eta_{0}: T M_{p} \rightarrow\left(T M_{p}\right)_{0}$ is the isomorphism of $T M_{p}$ onto its tangent space at 0 obtained by assigning to each $X \in T M_{p}$ the tangent vector $\bar{X} \in\left(T M_{p}\right)_{0}$ to the curve $t X$ at $t=0$, then

$$
\left(\exp _{(p, T, A)}\right)_{*}(\bar{X})=\text { tangent vector to the curve } \exp _{(p, T, A)}(t X) \text { at } t=0 .
$$

However

$$
\exp _{(p, T, A)}(t X)=\gamma_{(t X, T(A, t X, t X))}(1)=\gamma_{(X, T(A, X, X))}(t),
$$

which is a second order geodesic through $p$ with initial condition $\gamma^{\prime}(0)=X$. Thus

$$
\left(\exp _{(p, T, A)}\right)_{*}(\bar{X})=X
$$

which implies that $\left(\exp _{(p, T, A)}\right)_{*}$ is onto and hence an isomorphism. Using the inverse function theorem we may conclude that $\exp _{(p, T, A)}$ is a diffeomorphism of a neighborhood of 0 in $T M_{p}$ onto a neighborhood of $p$ in $M$. The second part of the theorem follows from the fact that $\gamma_{(X, 0)}(t)$ is a first order geodesic.

Remark. A canonical choice for $T(A, X, X)$ is $\tilde{R}(A, X) X$ where $\tilde{R}$ is the curvature tensor of the first order connection.

We may use Theorem 4.2 to obtain a family of normal coordinate systems at each $p \in M$. Choose a basis $\left\{e_{1}, \cdots, e_{n}\right\}$ of $T M_{p}$, and let $\left\{u^{1}, \cdots, u^{n}\right\}$ be the dual basis in $T M_{p}^{*}$. Then

$$
x^{i}=u^{i} \circ \exp _{(p, T, A)}^{-1}
$$

is a normal coordinate system on some neighborhood of $p$ in $M$. We note that each curve through $p$ with coordinates $x^{i}=\xi^{i} t$ is a second order geodesic with initial conditions $(X, A)$, where $X=\xi^{i} e_{i}$ for

$$
\exp _{(p, T, A)}(t X)=\gamma_{(t X, T(A, t X, t X))}(1)=\gamma_{(X, T(A, X, X))}(t) .
$$


Remark. If $A=a^{i} X_{i}^{v}$ relative to the basis $X_{i}^{v}$, then $\left(\Gamma_{i i}^{i}\right)_{0}=a^{i}$ in the normal coordinates.

\section{Higher order curvature}

If $D$ is the second order connection on $M$ induced from a linear (first order) connection $\tilde{D}$ on $M$, then for a $C^{\infty}$ section $A$ of the vector bundle ${ }_{0}^{2} \Pi:{ }^{2} M \rightarrow$ $M$ and a vector field $X$ on $M$ from (4.7) and (3.12) we see that

$$
D_{X} A=\left({ }_{0}^{1} \Pi_{*} D_{X} A, \tilde{D}\left(D_{X} A\right)\right)=\left(\tilde{D}_{X}^{1} \Pi_{*} A, \tilde{D}_{X} \tilde{D}(A)\right) \text {. }
$$

If $A$ and $B$ are $C^{\infty}$ sections of ${ }_{0}^{2} \Pi:{ }^{2} M \rightarrow M$, then we take

$$
\begin{aligned}
{[A, B] } & =\left(\left[{ }_{0}^{1} \Pi_{*} A,{ }_{0}^{1} \Pi_{*} B\right],[\tilde{D}(A), \tilde{D}(B)]\right), \\
D_{A} B & =\left(\tilde{D}_{{ }_{0}^{1} \Pi_{*} A}{ }_{0}^{1} \Pi_{*} B, \tilde{D}_{\tilde{D}(A)} \tilde{D}(B)\right) .
\end{aligned}
$$

We define a second order tensor $T_{s}^{r}$ on $M$ as a mapping of the product of $r$ copies of the module $\mathscr{D}$ of $C^{\infty}$ sections of the vector bundle ${ }_{0}^{2} \Pi:{ }^{2} M \rightarrow M$ over the ring $C^{\infty}(M)$ of $C^{\infty}$ functions on $M$, with $s$ copies of the dual $\mathscr{D}^{*}$ of $\mathscr{D}$, into $C^{\infty}(M)$ which is $C^{\infty}(M)$ linear in each variable. Then we define the second order torsion tensor

$$
\text { Tor }(A, B)=D_{A} B-D_{B} A-[A, B] .
$$

It follows from (5.2) that

$$
\begin{aligned}
\operatorname{Tor}(A, B) & =\left({ }_{0}^{1} \Pi_{*} \operatorname{Tor}(A, B), \tilde{D} \text { Tor }(A, B)\right) \\
& =\left(\widetilde{T} o r\left({ }_{0}^{1} \Pi_{*} A,{ }_{0}^{1} \Pi_{*} B\right), \text { Tor }(\tilde{D}(A), \tilde{D}(B))\right),
\end{aligned}
$$

where Tor is the torsion of $\tilde{D}$. Thus we see that Tor is a bilinear, over $C^{\infty}(M)$, map of $\mathscr{D} \times \mathscr{D}$ into $C^{\infty}(M)$ and hence a second order tensor on $M$. Thus we have the following theorem.

Theorem 5.1. Suppose that $D$ is the second order connection induced by a linear (first order) connection on $M$. Then Tor $=0$ if and only if Tor $=0$.

Definition. A second order Riemannian metric on $M$ is a positive definite inner product $\langle$,$\rangle on each fiber { }_{0}^{2} \Pi^{-1}(p)$ of the vector bundle ${ }_{0}^{2} \Pi:{ }^{2} M \rightarrow M$ having the property that if $A, B \in \mathscr{D}$, then the function defined by $\langle A, B\rangle_{p}=$ $\left\langle A_{p}, B_{p}\right\rangle$ belongs to $C^{\infty}(M)$.

If $\langle X, Y\rangle$ is a Riemannian metric (first order) on $M$, then it induces the second order Riemannian metric

$$
\langle A, B\rangle=\left\langle{ }_{0}^{1} \Pi_{*} A,{ }_{0}^{1} \Pi_{*} B\right\rangle+\langle\tilde{D}(A), \tilde{D}(B)\rangle
$$

on $M$, where $A, B \in \mathscr{D}$.

Definition. A second order linear connection on $M$ is the Riemannian connection associated with a given second order metric provided that 


$$
\text { Tor }(A, B)=0, \quad X\langle A, B\rangle=\left\langle D_{X} A, B\right\rangle+\left\langle A, D_{X} B\right\rangle,
$$

where $A, B \in \mathscr{D}$, and $X$ is a vector field on $M$.

Theorem 5.2. If $\tilde{D}$ is the linear (first order) Riemannian connection associated with a given (first order) metric $\langle$,$\rangle , then the second order con-$ nection $D$ on $M$ induced by $\tilde{D}$ is the Riemannian connection associated with the second order metric induced by $\langle$,$\rangle .$

Proof. That Tor $(A, B)=0$ follows from the fact that $\tilde{D}$ is Riemannian and Theorem 5.1. Since

$$
X\langle A, B\rangle=X\left\langle{ }_{0}^{1} \Pi_{*} A,{ }_{0}^{1} \Pi_{*} B\right\rangle+X\langle\tilde{D}(A), \tilde{D}(B)\rangle,
$$

and $\tilde{D}$ is Riemannian with respect to the metric on the right, we have

$$
\begin{aligned}
X\langle A, B\rangle= & \left\langle\tilde{D}_{X_{0}^{1}} \Pi_{*} A,{ }_{0}^{1} \Pi_{*} B\right\rangle+\left\langle{ }_{0}^{1} \Pi_{*} A, \tilde{D}_{X_{0}}^{1} \Pi_{*} B\right\rangle \\
& +\left\langle\tilde{D}_{X} \tilde{D}(A), \tilde{D}(B)\right\rangle+\left\langle\tilde{D}(A), \tilde{D}_{X} \tilde{D}(B)\right\rangle .
\end{aligned}
$$

Using (5.1) and (5.5) we have

$$
X\langle A, B\rangle=\left\langle D_{X} A, B\right\rangle+\left\langle A, D_{X} B\right\rangle .
$$

We take

$$
R(X, Y) A=D_{X} D_{Y} A-D_{Y} D_{X} A-D_{[X, Y]} A,
$$

where $X, Y$ are vector fields on $M, A \in \mathscr{D}$, and $D$ is a linear second order connection on $M$. If $D$ is the second order connection induced by a linear (first order) connection, then from (5.1)

$$
D_{X} D_{Y} A=\left({ }_{0}^{1} \Pi_{*} D_{X} D_{Y} A, \tilde{D} D_{X} D_{Y} A\right)=\left(\tilde{D}_{X} \tilde{D}_{Y}^{1} \Pi_{*} A, \tilde{D}_{X} \tilde{D}_{Y} \tilde{D}(A)\right),
$$

and so we see that

$$
\begin{aligned}
R(X, Y) A & \left.={ }_{0}^{1} \Pi_{*} R(X, Y) A, \tilde{D} R(X, Y) A\right) \\
& =\left(\tilde{R}(X, Y)_{0}^{1} \Pi_{*} A, \tilde{R}(X, Y) \tilde{D}(A)\right) .
\end{aligned}
$$

If we take

$$
R(A, B, X, Y)=\langle A, R(X, Y) B\rangle,
$$

where $A, B \in \mathscr{D}$, and $X, Y$ are vector fields, then we have

Theorem 5.3. If $D$ is Riemannian with respect to $\langle$,$\rangle , then$

$$
\begin{aligned}
& R(A, B, X, Y)=-R(A, B, Y, X), \\
& R(A, B, X, Y)=-R(B, A, X, Y) .
\end{aligned}
$$


Proof. The first of (5.10) follows from the antisymmetry $R(X, Y)=$ $-R(Y, X)$, and the second from the parallelism of $D$ with respect to $\langle$,$\rangle by$ noting that

$$
\begin{aligned}
X Y\langle A, B\rangle= & \left\langle D_{X} D_{Y} A, B\right\rangle+\left\langle D_{X} A, D_{Y} B\right\rangle+\left\langle D_{Y} A, D_{X} B\right\rangle \\
& +\left\langle A, D_{X} D_{Y} B\right\rangle, \\
{[X, Y]\langle A, B\rangle=} & \left\langle D_{[X, Y]} A, B\right\rangle+\left\langle A, D_{[X, Y]} B\right\rangle
\end{aligned}
$$

for vector fields $X, Y$ and $A, B \in \mathscr{D}$, and hence that

$$
\langle R(X, Y) A, B\rangle+\langle A, R(X, Y) B)\rangle=0 .
$$

We may now define the two second order curvature tensors of a second order connection $D$ induced from a linear (first order) connection $\tilde{D}$ :

$$
\begin{aligned}
R_{\mathrm{I}}(C, E) A & =\left(\tilde{R}\left({ }_{0}^{1} \Pi_{*} C,{ }_{0}^{1} \Pi_{*} E\right)_{0}^{1} \Pi_{*} A, \tilde{R}\left({ }_{0}^{1} \Pi_{*} C,{ }_{0}^{1} \Pi_{*} E\right) \tilde{D}(A)\right), \\
R_{\mathrm{II}}(C, E) A & =\left(\tilde{R}(\tilde{D}(C), \tilde{D}(E))_{0}^{1} \Pi_{*} A, \tilde{R}(\tilde{D}(C), \tilde{D}(E)) \tilde{D}(A)\right)
\end{aligned}
$$

and in the case where $D$ is Riemannian with respect to $\langle$,$\rangle we define the$ second order Riemann-Christoffel tensors

$$
\begin{aligned}
R_{\mathrm{I}}(A, B, C, D) & =\left\langle A, R_{\mathrm{I}}(C, D) B\right\rangle, \\
R_{\mathrm{II}}(A, B, C, D) & =\left\langle A, R_{\mathrm{II}}(C, D) B\right\rangle .
\end{aligned}
$$

If $G(A, B)=\langle A, A\rangle\langle B, B\rangle-\langle A, B\rangle^{2} \neq 0$, then we define

$$
\begin{aligned}
K_{\mathrm{I}}(A, B) & =R_{\mathrm{I}}(A, B, A, B) / G(A, B), \\
K_{\mathrm{II}}(A, B) & =R_{\mathrm{II}}(A, B, A, B) / G(A, B) .
\end{aligned}
$$

Upon taking $X={ }_{0}^{1} \Pi_{*} A, Y={ }_{0}^{1} \Pi_{*} B$ in $R_{\mathrm{I}}$ and $X=\tilde{D}(A), Y=\tilde{D}(B)$ in $R_{\mathrm{II}}$ of (3.12) it follows from (5.10) that $K_{i}$, for $i=\mathrm{I}$, II, has the property

$$
K_{i}(A, B)=K_{i}(B, A)=K_{i}(r A, s B)=K_{i}(A+t B, B) .
$$

Thus, if $G(A, B) \neq 0$, and $A, B$ are linearly independent (and $a d-c b \neq 0$ ), then

$$
K_{i}(A, B)=K_{i}(a A+b B, c A+d B),
$$

and we have

Theorem 5.4. For each point $p \in M$ let $A, B \in{ }^{2} M_{p}$. Then the scalars $K_{\mathrm{I}}(A, B)$ and $K_{\mathrm{II}}(A, B)$ depend only upon the subspace of ${ }^{2} M_{p}$ spanned by $A$ and $B$. We call $K_{\mathrm{I}}$ and $K_{\mathrm{II}}$ the Ist and IInd second order curvatures of $M$ respectively. 
Remark. If ${ }_{0}^{1} \Pi_{*} A=X, \tilde{D}(A)=0,{ }_{0}^{1} \Pi_{*} B=Y, \tilde{D}(B)=0$, then $K_{\mathrm{I}}(A, B)$ $=K(X, Y)$ where $K$ is the usual (first order) curvature of $\tilde{D}$.

\section{References}

[ 1 ] R. H. Bowman, On differentiable extensions, Tensor, N. S. 21 (1970) 139-150.

[2] Tong Van Duc, Connexions et structures particulières sur les fibers vectoriels, C. R. Acad. Sci. Paris 270 (1970) 661-664.

[ 3 ] J. Vilms, Connections on tangent bundles, J. Differential Geometry 1 (1967) 235243.

[ 4 ] K. Yano \& S. Ishihara, Differential geometry of tangent bundles of order 2, Kōdai Math. Sem. Rep. 20 (1968) 318-354.

[ 5 ] K. Yano \& S. Kobayashi, Prolongations of tensor fields and connections to tangent bundles. I, J. Math. Soc. Japan 18 (1966) 194-210.

[6] P. Dombrowski, Geometry of the tangent bundle, J. Reine Angew. Math. 210 (1962) 73-88.

Arkansas State University 
